\title{
Microstructural studies of bulk and thin film GDC
}

\author{
K. Muthukkumaran • P. Kuppusami • \\ R. Kesavamoorthy $\cdot$ T. Mathews $\cdot$ E. Mohandas • \\ V. S. Raghunathan $\cdot$ S. Selladurai
}

Received: 26 January 2007 / Revised: 31 July 2007 / Accepted: 2 August 2007 / Published online: 1 September 2007

(C) Springer-Verlag 2007

\begin{abstract}
Ceria rare earth solid solutions are known as solid electrolyte with potential application in oxygen sensors and solid oxide fuel cells. We report the preparation of gadolinia-doped ceria, $\mathrm{Ce}_{0.90} \mathrm{Gd}_{0.10} \mathrm{O}_{1.95}$, by the conventional solid-state reaction method and the preparation of thin films from a sintered pellet of gadolinia-doped ceria by the pulsed laser deposition technique. The effect of process conditions, such as substrate temperature, oxygen partial pressure, and laser energy on microstructural properties of these films are examined using powder X-ray diffraction, scanning electron microscopy, atomic force microscopy, and Raman spectroscopy.
\end{abstract}

Keywords Doped ceria · Thin films ·

Pulsed laser deposition · Microstructure .

Atomic force microscopy $\cdot$ Raman spectroscopy

K. Muthukkumaran $\cdot$ S. Selladurai

Department of Physics, Anna University,

Chennai 600 025, Tamilnadu, India

P. Kuppusami • E. Mohandas • V. S. Raghunathan

Material Characterization Group,

Indira Gandhi Centre for Atomic Research,

Kalpakkam 603 102, Tamilnadu, India

R. Kesavamoorthy $\cdot$ T. Mathews

Materials Science Division,

Indira Gandhi Centre for Atomic Research,

Kalpakkam 603102, Tamilnadu, India

Present address:

K. Muthukkumaran $(\triangle)$

Max-Plank Institute für Metallforschung,

Hesenbergstrße 3,

70569, Stuttgart, Germany

e-mail: muthuk24@yahoo.co.in

\section{Introduction}

The solid oxide fuel cell (SOFC) offers an environmentally friendly alternative to conventional energy conversion devices [1]. A typical SOFC consists of two electrodes (anode and cathode) separated by an electrolyte. Yttria-stabilized zirconia (YSZ) is considered to be the most reliable candidate as the electrolyte and has been widely used. However, for the YSZ to acquire sufficiently high oxygen ion conductivity, a high operating temperature, typically around $1,223 \mathrm{~K}$, is required, which severely limits the development of SOFCs [2]. Therefore, it is necessary to lower the operating temperature to the range $773-973 \mathrm{~K}$. The lower temperature of application provides a greater flexibility in the choice of electrode and interconnects materials, reduction in thermal stresses in active ceramic structures, as well as a longer lifetime of the cells. The principal approach to obtain the required reduction in the operating temperature is to combine the thin film fabrication technique with the employment of electrolyte materials having higher oxide ion conductivity [3]. Concerted efforts are being made by researchers around the world to develop such materials. Alternative electrolytes currently being investigated involve doped $\mathrm{CeO}_{2}$ [4] and ( $\mathrm{Sr}, \mathrm{Mg}$ )-doped $\mathrm{LaGaO}_{3}$ [5].

Pure cerium oxide is basically not a fast oxygen ion conductor unless it is doped with aliovalent cations, especially trivalent rare earth ions. The introduction of aliovalent cations to the host lattice can increase the oxygen vacancy concentration and concomitantly improve oxide ion conductivity in cerium oxide. $\mathrm{Y}_{2} \mathrm{O}_{3}, \mathrm{Gd}_{2} \mathrm{O}_{3}$, and $\mathrm{Sm}_{2} \mathrm{O}_{3}$ are the usual dopants, which can significantly enhance the ionic conductivity of ceria [6]. Gadolinia-doped ceria (GDC) has given much attention because of its great potential application as the electrolyte in intermediate tem- 
Table 1 Typical deposition parameters

\begin{tabular}{ll}
\hline Parameter & Value \\
\hline Laser & KrF excimer $(248 \mathrm{~nm})$ \\
Pulse duration & $30 \mathrm{~ns}$ \\
Repetition rate & $10 \mathrm{~Hz}$ \\
Laser energy & $100-600 \mathrm{~mJ} /$ pulse \\
Energy density & $2-5 \mathrm{~J} / \mathrm{cm}^{2}$ \\
Target & $\mathrm{Sintered} 10 \mathrm{~mol} \%$ Gd-doped $\mathrm{CeO}_{2}$ \\
Substrate & $\mathrm{Si}(111)$ \\
Substrate temperature & $673-773 \mathrm{~K}$ \\
Target-substrate spacing & $45 \mathrm{~mm}$ \\
Base pressure & $\sim 3.5 \times 10^{-5} \mathrm{mbar}$ \\
\hline
\end{tabular}

perature SOFCs [7] The addition of gadolinia into ceria can be written in Kroger-Vink notation [8] as:

$$
\mathrm{Gd}_{2} \mathrm{O}_{3} \rightarrow 2 \mathrm{Gd}_{\mathrm{Ce}}^{\prime}+3 \mathrm{O}_{0}^{\mathrm{x}}+\mathrm{V}_{0}^{*}
$$

In this paper, preparation of sintered pellets of GDC $\mathrm{Ce}_{0.90}$ $\mathrm{Gd}_{0.10} \mathrm{O}_{1.95}$, and fabrication of $\mathrm{Ce}_{0.90} \mathrm{Gd}_{0.10} \mathrm{O}_{1.95}$ thin films by pulsed laser deposition technique [9] are presented. The influences of substrate temperature, oxygen partial pressure, and laser energy on crystal structure and film morphology are investigated. These parameters are known to be very important to obtain good-quality films for SOFC and sensor applications.

\section{Experimental procedures}

Samples corresponding to the formula $\mathrm{Ce}_{0.90} \mathrm{Gd}_{0.10} \mathrm{O}_{1.95}$ (GDC) were prepared from ceria and gadolinia by the conventional solid-state reaction technique. $\mathrm{CeO}_{2}(99.99 \%$ pure) mixed with appropriate quantity of $\mathrm{Gd}_{2} \mathrm{O}_{3}(99.99 \%$ pure) was ground in a mortar and pestle for $18 \mathrm{~h}$. About $5 \mathrm{~g}$ of the powder was uniaxially pressed into pellets of $20 \mathrm{~mm}$ diameter and $6 \mathrm{~mm}$ thickness at a pressure of $4 \mathrm{ton} / \mathrm{in.}{ }^{2}$. These pellets were sintered at $1,823 \mathrm{~K}$ for $6 \mathrm{~h}$ in air [10]. To examine the microstructure of the sintered pellet, the sample was metallographically polished with diamond paste grit size of $0.5 \mu \mathrm{m}$ and etched using a solution containing a molar ratio of $1: 2 \mathrm{HCl}$ and $\mathrm{HNO}_{3}$ for $30 \mathrm{~min}$ at room temperature.

The deposition of GDC films was carried out using a $\mathrm{KrF}$ excimer laser of 248-nm wavelength (Compex 205 from Lambda Physik) and a turbo-pumped deposition system [11-13] with typical laser repetition rate of $10 \mathrm{~Hz}$ using laser energy in the range $200-600 \mathrm{~mJ} /$ pulse. Substrates of Si (111) orientation of size $10 \times 10 \mathrm{~mm}$ were used as the substrate. In this experiment, the excimer laser was focused onto the GDC target at an angle of incidence of $45^{\circ}$. During deposition, the target was rotated and oscillated to avoid pitting. The deposition parameters used are given in Table 1.

The thickness of the deposited films was measured using a 3010 Dektak profilometer. The phase identification and grain size estimation were carried out using a Philips PW1730 $\mathrm{X}$-ray diffractometer $(\mathrm{XRD})$ system. The grain size, $d_{\mathrm{g}}$ was determined using the Scherrer formula:

$d_{g}=k \cdot \lambda / \cos \theta \cdot \sqrt{\left(B^{2}-b^{2}\right)}$

where $\lambda$ is the wavelength $(0.15418 \mathrm{~nm}), k$ is the correction factor $(k=0.97), \theta$ is the diffraction angle, $B$ is the full width at half maximum (FWHM) of the (200) diffraction line of the film, and $b$ is the FWHM of the instrumental broadening profile. A value of $b=0.175^{\circ}$ determined from the (200) reflection of an annealed powder sample of pure $\mathrm{CeO}_{2}$ was used. The values of $B$ and $b$ were measured on the $2 \theta$ scales. Surface morphology and composition were analyzed using an XL30 ESEM Philips scanning electron microscope (SEM) fitted with an X-ray energy-dispersive analyzer.

Atomic force microscopy (AFM) images of thin films were taken in the tapping mode (DFM mode using SPA400, Seiko Instruments). The root mean square roughness and grain sizes were also obtained from the images. The Raman spectra of the films were recorded using a 488-nm line of an argon ion laser (Coherent USA) in the backscattering geometry at room temperature. A Raman spectrometer built with a double-grating monochromator SPEX model 14018 was used. A $30-\mathrm{mW}$ incident laser beam was focused on the sample to a spot size of about $100-\mu \mathrm{m}$ diameters. The spectral resolution of the monochromator was $4.2 \mathrm{~cm}^{-1}$. The spectra were recorded digitally using a microprocessorbased automated data collection system with a step of $1 \mathrm{~cm}^{-1}$ and a collection time of $10 \mathrm{~s}$.

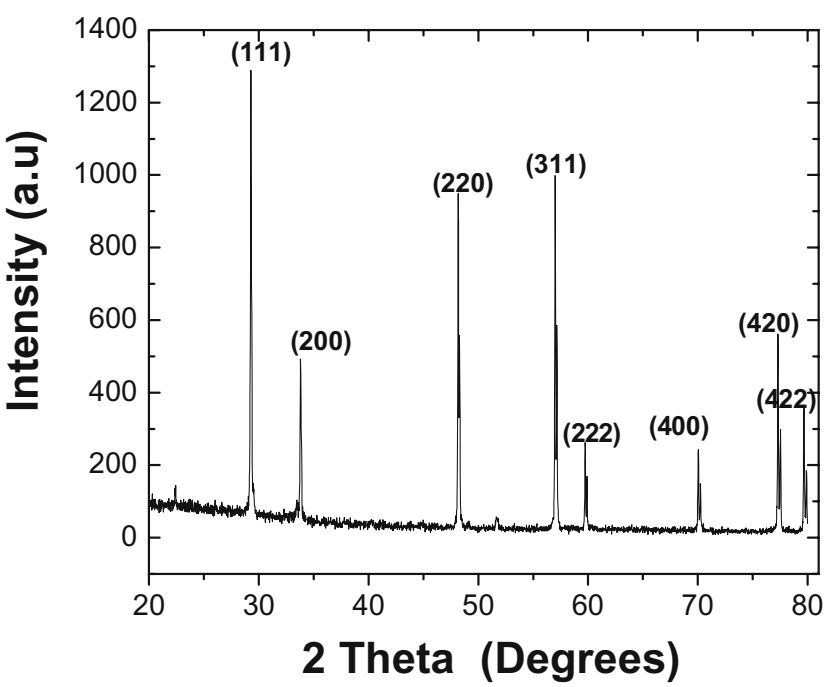

Fig. 1 Powder XRD pattern of $\mathrm{Ce}_{0.9} \mathrm{Gd}_{0.1} \mathrm{O}_{1.95}$ bulk sintered target material 
Fig. 2 Microstructure of the sintered GDC pellet. a Optical microscopy and b SEM
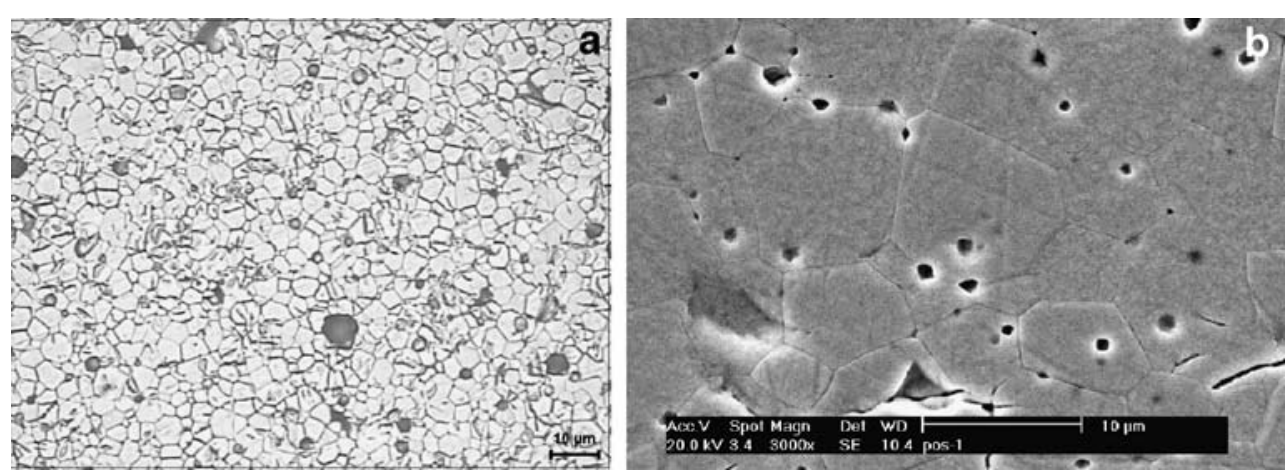

\section{Results and discussion}

Microstructure of the sintered pellet

Figure 1 shows the powder XRD pattern for the GDC sample containing $10 \mathrm{~mol} \%$ of gadolinia sintered at $1,823 \mathrm{~K}$ for $6 \mathrm{~h}$ [10]. The XRD pattern revealed no secondary or impurity phases. The data on peak positions, peak widths, and relative peak intensity were analyzed using a peak-fitting program, which calculated a theoretical data curve from the original data assuming a symmetrical peak shape. Lattice parameters were then refined from the fitted data using a least square fitting procedure with an error of about $1 \%$. The lattice parameter was determined to be $0.544 \mathrm{~nm}$ and is slightly higher than that of undoped ceria [10]. This increase in the lattice parameter has been attributed to the slightly larger radius of the dopant $\mathrm{Gd}^{3+}(0.097 \mathrm{~nm})$ compared to that of the host ion $\mathrm{Ce}^{4+}(0.090 \mathrm{~nm})$. It is observed that for the given doping concentration of gadolinia, the sintering temperature of $1,823 \mathrm{~K}$ is adequate to ensure the formation of a solid solution.

The density of the sintered specimens was determined by the Archimedes method using dibutyl phthalate as the medium. All the samples used in the present study had a density of about $90 \%$ of the theoretical value.

The microstructure of the GDC pellet was investigated by means of optical microscopy and scanning electron microscopy. Figure 2a,b shows the optical and scanning electron micrographs of GDC pellet sintered at $1,823 \mathrm{~K}$. The microstructures show that the target is well sintered, and they contain grain size of about 5-8 $\mu \mathrm{m}$. Except a few pores, the grain boundaries do not contain any secondary phases.

\section{Thin films}

Thin films of $\mathrm{Ce}_{0.90} \mathrm{Gd}_{0.10} \mathrm{O}_{1.95}$ were prepared at different substrate temperature, oxygen partial pressure, and laser energy. To study the influence of substrate temperature on the film growth, all the films were deposited at $200 \mathrm{~mJ} /$ pulse for $1 \mathrm{~h}$. The typical thickness of the films deposited on $\mathrm{Si}$ (111) substrates under the above deposition condition was about $500 \mathrm{~nm}$. Figure 3 shows the SEM picture of the film deposited at $873 \mathrm{~K}$ showing a featureless structure. It is interesting to note that the microstructure is free from cracks, and pores and droplets of coarser particles were rarely noted on the surface of the films. From the XRD patterns shown in Fig. $4 a$, it is seen that the films are polycrystalline in the temperature range of deposition. The crystallinity of the films has been found to increase with increasing substrate temperature. Because the silicon substrate with $<111>$ orientation has a close matching with GDC, the substrate peaks (X) are found to match with GDC for (111) and (222) reflections. The lattice parameter of the films is found to increase gradually from 0.540 to $0.548 \mathrm{~nm}$ in the temperature range $473-973 \mathrm{~K}$. Figure $4 \mathrm{~b}$ shows that at $873 \mathrm{~K}$, the lattice parameter of the film reaches the bulk lattice parameter value of $0.544 \mathrm{~nm}$ [10]. Figure $4 \mathrm{c}$ shows the effect of substrate temperature on the grain size. The grain size is found to increase from $\sim 15$ to $\sim 20 \mathrm{~nm}$ owing to increased mobility of adatoms with increasing substrate temperature. At $973 \mathrm{~K}$, peak broadening and loss of intensity of the diffraction peaks are noticed. This could be attributed to the interfacial reaction of silicon with the films at the higher deposition temperature.

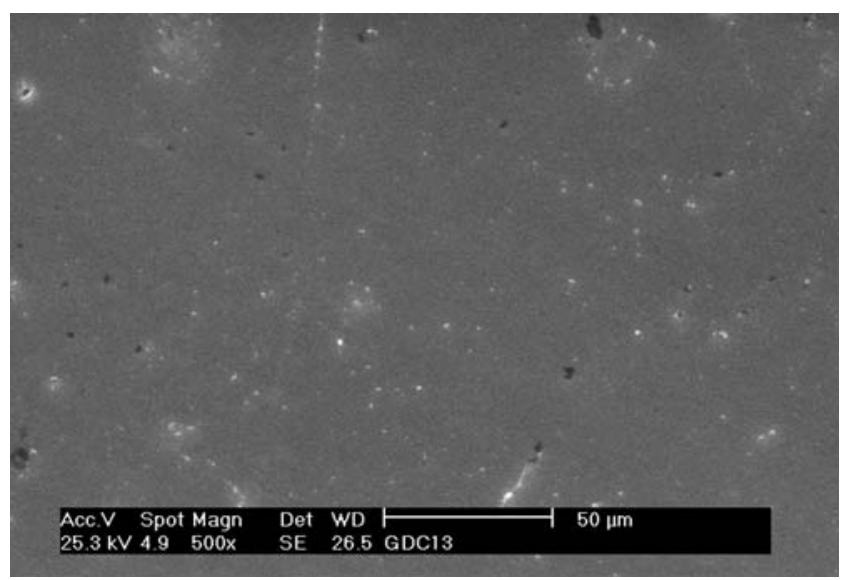

Fig. 3 SEM picture film deposited at $873 \mathrm{~K}$ 

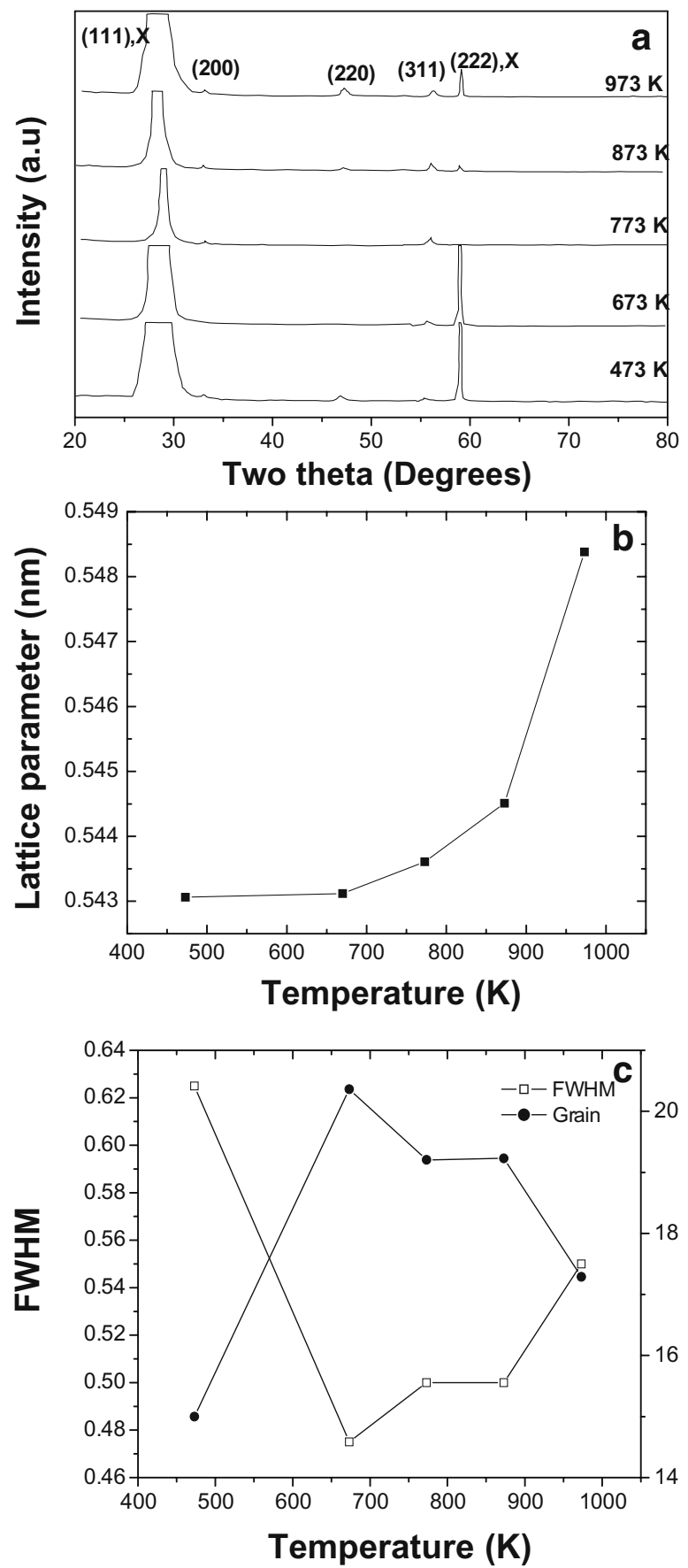

Fig. 4 a XRD pattern of $\mathrm{Ce}_{0.90} \mathrm{Gd}_{0.10} \mathrm{O}_{1.95}$ films at various substrate temperature. b Lattice parameter as function of substrate temperature. c Grain size as function of substrate temperature

To study the effect of ambient oxygen partial pressure, thin film deposition at oxygen partial pressures in the range of $5 \times 10^{-5}$ to 0.3 mbar were carried out at a laser energy of $200 \mathrm{~mJ} /$ pulse at $873 \mathrm{~K}$ for $1 \mathrm{~h}$. XRD results shown in Fig. 5a indicate a significant influence of the oxygen partial pressure on the growth characteristics of the films. Figure $5 \mathrm{~b}$ shows the trend in the orientation of the film as a function of oxygen partial pressure. At $5 \times 10^{-5}$ mbar base pressure, the films are weakly crystalline and have (111) orientation. With increasing oxygen partial pressure from 0.01 to $0.2 \mathrm{mbar}$, the films are (200) oriented. At 0.3 mbar of oxygen partial pressure, there is a clear growth of polycrystalline films of GDC. These experiments clearly demonstrate that the films of desired orientation could be obtained by appropriate selection of oxygen partial pressure.

To study the influence of laser energy on the growth of doped ceria on $\mathrm{Si}$ (111) substrates, deposition was carried out in the energy range $200-600 \mathrm{~mJ} /$ pulse at a substrate temperature of $873 \mathrm{~K}$ for $1 \mathrm{~h}$ at a base pressure of $5 \times 10^{-5} \mathrm{mbar}$. The thickness and the deposition rate were found to increase significantly with laser energy. For instance, the deposition rate has increased from $0.02 \mathrm{~nm} / \mathrm{s}$ at $200 \mathrm{~mJ} /$ pulse to $0.2 \mathrm{~nm} / \mathrm{s}$ at $600 \mathrm{~mJ} /$ pulse; this was explained in our earlier studies [14]. Figure 6a shows the XRD pattern of GDC films grown at various energy levels on silicon substrate. The patterns indicate that films are single phasic with no detectable secondary phases. The patterns were indexed
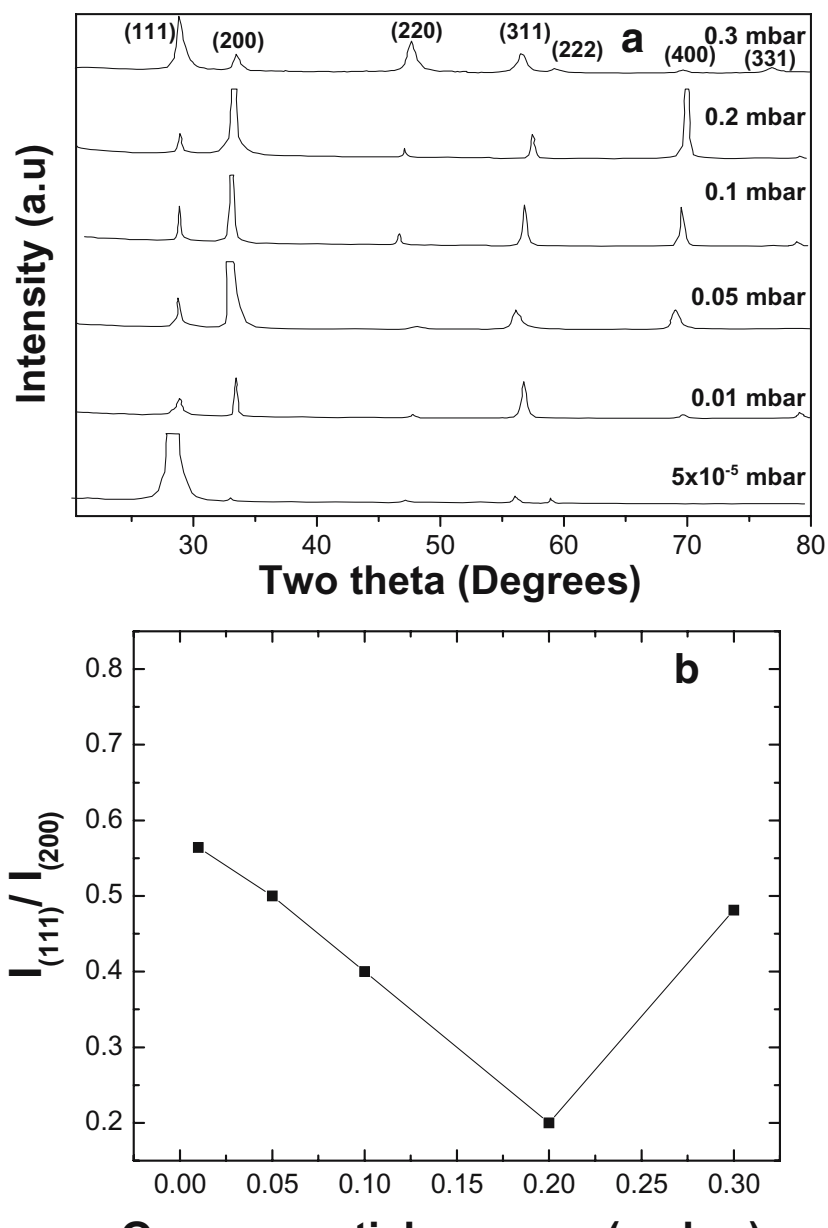

Oxygen partial pressure( $\mathrm{m}$ bar)

Fig. 5 a Influence of oxygen partial pressure $\mathrm{Ce}_{0.90} \mathrm{Gd}_{0.10} \mathrm{O}_{1.95}$ films on $\mathrm{Si}(111)$. b Intensity ratio $\mathrm{I}(111) / \mathrm{I}(200)$ as function oxygen partial pressure 

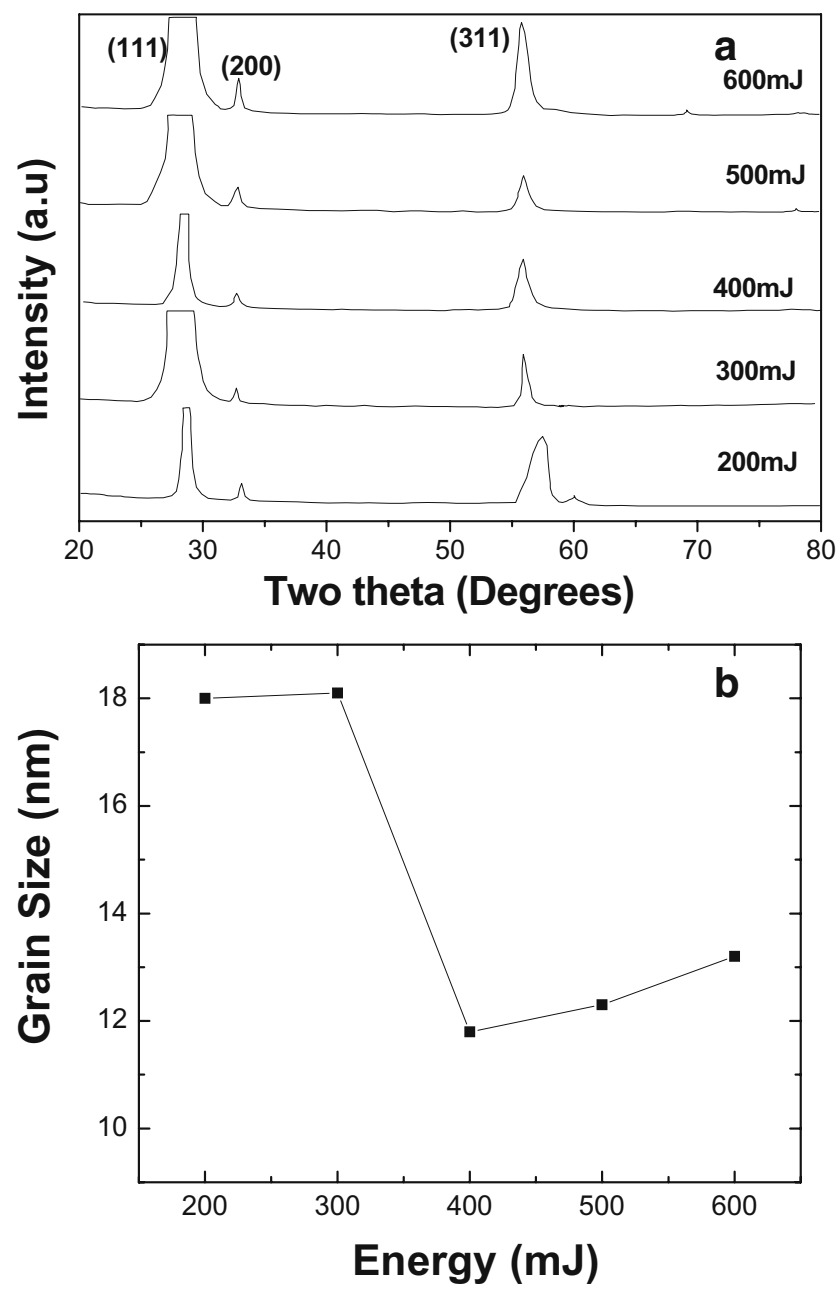

Fig. 6 a XRD pattern showing influence of laser energy. b Grain size as function of laser energy

as $\mathrm{CeO}_{2}$ with fluorite structure. Figure $6 \mathrm{~b}$ shows that the grain size decreases from 18 to $13 \mathrm{~nm}$ at higher energy. It is possible that with increasing laser energy, the deposition rate increases rapidly, which may reduce adatom mobility resulting in the reduction in the grain size.

AFM was used to study the surface morphology of the films deposited at various laser energy. Typical examples of AFM 3D images of films grown at various energies are shown in Fig. 7. AFM images largely indicate a fine distribution of globular grains of 60-70-nm sizes along with a few randomly distributed conical-shaped grains in the energy range $200-400 \mathrm{~mJ} /$ pulse. These grains are found to be coarsened $(\sim 150 \mathrm{~nm})$ and tilted toward the substrate surface in the energy range $500-600 \mathrm{~mJ} /$ pulse. With the difference in grain size accordance with XRD, it is assumed that the AFM gives only local surface area information not in the crystal plane. AFM examination at higher magnification also reveals several smaller grains within larger grains. Further, the increase in the grain size has increased the surface roughness from 0.75 to $1.5 \mathrm{~nm}$. The shapes of the grains are clearly indicative of an island growth mechanism operating in these laser-ablated GDC thin films [15].

Figure 8a shows the Raman spectra of the nanocrystalline thin films of $\mathrm{Ce}_{0.90} \mathrm{Gd}_{0.10} \mathrm{O}_{1.95}$ prepared at various substrate temperatures using a laser energy of $200 \mathrm{~mJ} /$ pulse at a base pressure of $3.5 \times 10^{-5}$ mbar. Figure $8 \mathrm{~b}$ shows the Raman shift and FWHM of the Raman spectra shown in Fig. 9a. The Raman-active mode in this material corresponds to a frequency of $\omega_{\mathrm{R}} \sim 466 \mathrm{~cm}^{-1}$, which is attributed to a symmetrical stretching mode of the $\mathrm{Ce}-\mathrm{O} 8$ vibrational unit of cubic ceria $[16,17]$. Therefore, this mode should be very sensitive to any disorder in the oxygen sublattice resulting from thermal and/or grain size induced nonstoichiometry. The influence of the microstructure on the shape of the Raman spectrum was observed by the Raman shift and the width of the Raman mode [18-23]. Figure 8 shows the variation in FWHM and Raman line shift as a function of substrate temperature. FWHM decreases with increasing substrate temperature, which might be due to the decrease in defect concentration as a result of reduction in intergranular volume fraction because of the increase in the grain size in agreement with the XRD analysis. As the substrate temperature increases, oxygen desorption from the film during deposition at the base pressure of $3.5 \times 10^{-5} \mathrm{mbar}$ would increase resulting in increased oxygen nonstoichiometry. This may lead to a slight increase in the $\mathrm{Ce}-\mathrm{O}$ and $\mathrm{Gd}-\mathrm{O}$ bond lengths, which would result in a Raman peak red shift as observed in Fig. 9.

Figure 9a illustrates the Raman spectra of GDC films obtained at $873 \mathrm{~K}$ as a function of laser energy. These films were deposited in the base pressure of $3 \times 10^{-5}$ mbar. Like the effect of substrate temperature, laser energy also shows a significant shift in the Raman line and FWHM when the laser energy is increased from 200 to $600 \mathrm{~mJ} / \mathrm{pulse}$ (Fig. 9b). When $\mathrm{Gd}$ replaces Ce at some lattice sites, the Raman line is expected to red shift because of heavier mass (Gd) replacing lighter mass (Ce). This red shift depends on the fraction of the substitution. As the laser energy increases, the fraction of $\mathrm{Gd}$ incorporated in the film seems to be increasing as suggested by the Raman red shift shown in Fig. 9. In contrast, FWHM increases with laser energy and saturates beyond $300 \mathrm{~mJ} /$ pulse indicating that there is a rapid increase in the defect concentration initially, and it does not increase significantly. Our recent observation of GDC thin films using high-resolution electron microscopy also reveal defects like dislocations, ledges, bending of lattices, etc. for the films grown with laser energy exceeding $300 \mathrm{~mJ} /$ pulse [14] in agreement with the Raman spectra. The present study qualitatively suggests that substrate temperature and laser energy significantly influences the microstructure of the films, and appropriate selections of the process parameters are required to achieve GDC films with desired properties for technological applications. 
Fig. 7 Typical AFM images recorded at different laser energy a 200 , b 400 , c 500 , and d $600 \mathrm{~mJ} / \mathrm{pulse}$

Fig. 8 a Laser Raman spectra of $\mathrm{Ce}_{0.90} \mathrm{Gd}_{0.10} \mathrm{O}_{1.95}$ films prepared at different substrate temperature. b Influence of laser energy at a substrate temperature of $873 \mathrm{~K}$
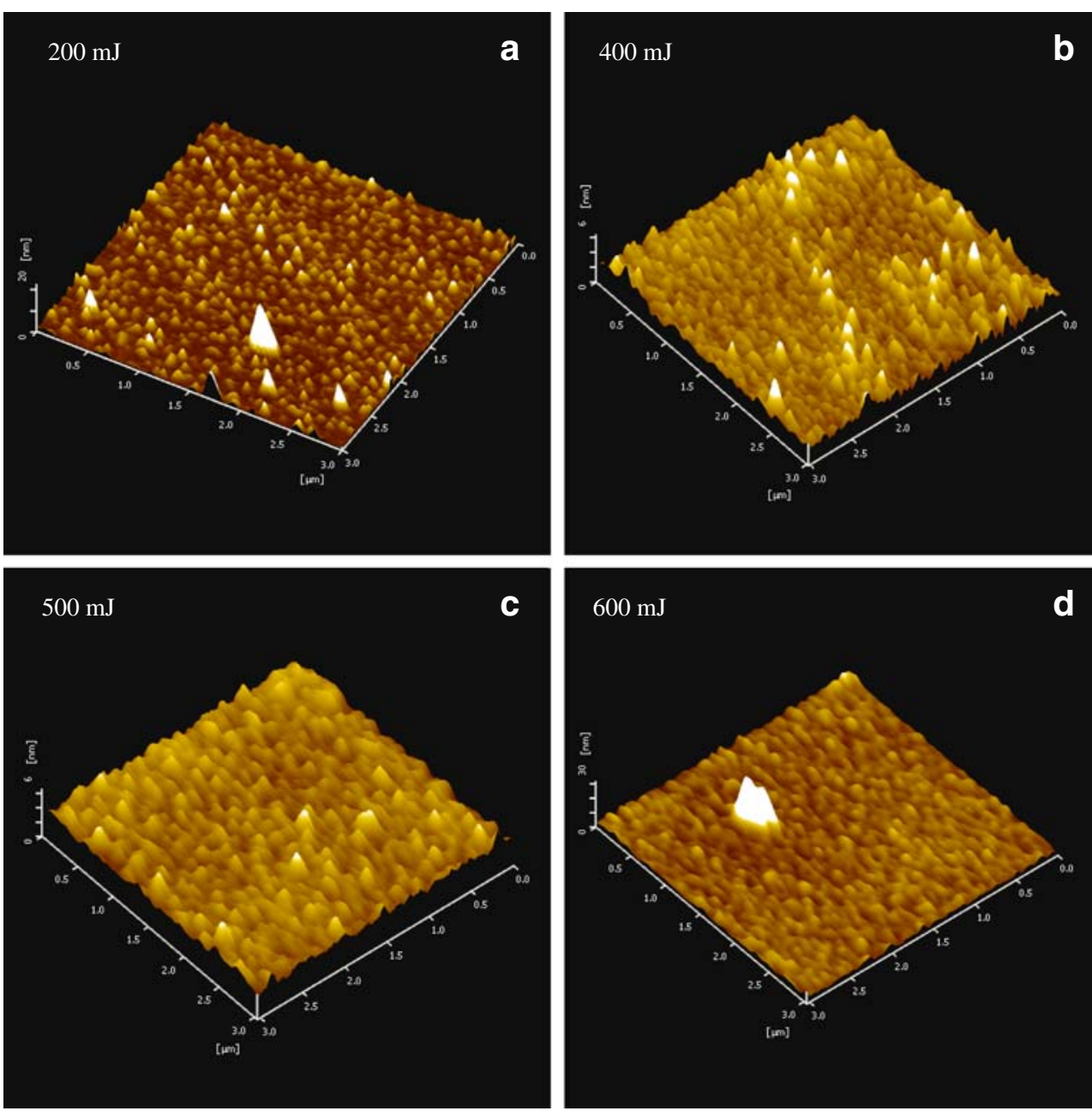

d
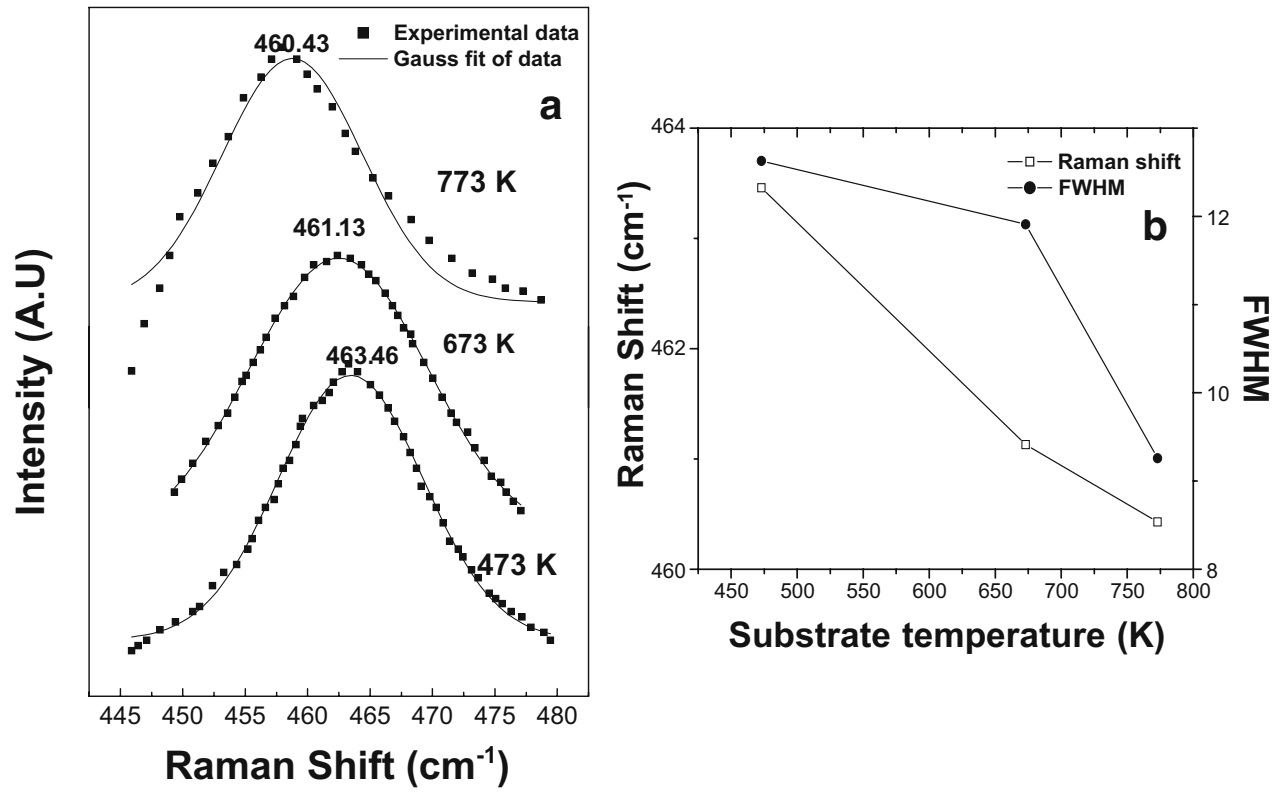

Substrate temperature (K) 
Fig. 9 Raman shift and FWHM of at various a substrate temperatures and $\mathbf{b}$ laser energies

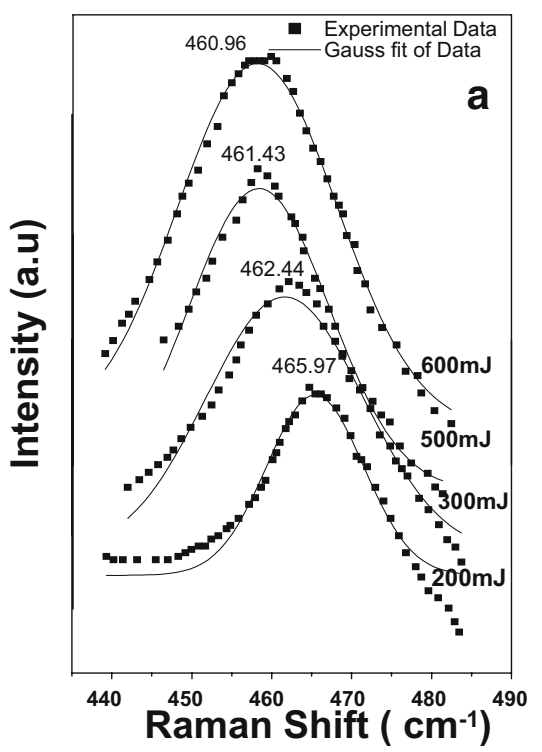

\section{Conclusions}

(1) Sintered pellet of $\mathrm{Ce}_{0.90} \mathrm{Gd}_{0.10} \mathrm{O}_{1.95}$ was synthesized by a solid-state reaction route, and then the sintered pellet was used as a target for the fabrication of thin films by pulsed laser ablation.

(2) The influence of the substrate temperature, oxygen partial pressures, and laser energy on the growth characteristics of GDC was studied. The films are mostly polycrystalline, and grain size increases with increasing substrate temperature. Oxygen addition during the growth of the film has been found to alter the film orientation from (111) to (200) in the partial pressure range $0.1-0.2$ mbar. In addition to increase in the deposition rate, the laser energy has significantly modified the surface morphology of the films. The results obtained from the Raman spectra indicate that both the substrate temperature and laser energy could influence the defect concentration in the GDC films grown by pulsed laser ablation.

Acknowledgments The authors acknowledge the help of Mrs. M. Jyothi for the XRD analysis and Dr. P. Parameswaran for SEM examination. They also express sincere thanks to Dr. M. Vijayalakshmi, Head of Physical Metallurgy Section, Dr. P. R. Vasudeva Rao, Director of Metallurgy and Materials Group, and Dr. Baldev Raj, Director of IGCAR, for their support and encouragement.

\section{References}

1. Doshi CR, Richards VI, Cartex JD, Way XP, Krumpett M (1999) J Electrochem Soc 146(4):1273-1278

2. Steel BCH (2000) Solid State Ion 129(1-4):95-11
3. Ishihara T, Sato K, Takita Y (1996) J Am Ceram Soc 79(4):913-919

4. Huang W, Shuk P, Greenblatt M (1997) Solid State Ion 100(1-2):23

5. Mizusaki J, Mori N, Takai H, Yonemura Y, Minamiue H, Tagawa H, Dokiya M, Inaba H, Naraya K, Sasamoto T, Hashimoto T (2000) Solid State Ion 129(1-4):163-177

6. Kilner JA (2000) Solid State Ion 129(1-4):13-23

7. Kilner JA, Brook RJ (1982) Solid State Ion 6(3):237-252

8. Kroger F, Vink HJ (1965) In: Seitz F, Turnbull D(eds) Solid state physics 305 cademic, New York

9. Kuppusami P, Padi SN, Muthukkumaran K, Mohandas E, Raghunathan VS (2005) Surf Eng 21(3):172-175

10. Muthukkumaran K, Kuppusami P, Mohandas E, Raghunathan VS, Selladurai S (2004) International Symposium of Research Students on Materials Science and Engineering December 20-22, Chennai, India

11. Padhi SN, Muthukkumaran K, Kuppusami P, Mohandas E, Raghunathan VS, Hebbar KR (2004) International Symposium of Research Students on Materials Science and Engineering December 20-22, Chennai, India

12. Foschini CR, Souza DPF, Paulin Filho PI, Varela JA (2001) J Eur Ceram Soc 21(9):1143-1150

13. Mogensen M, Sammes NM, Tompsett GA (2000) Solid State Ion 129(1-4):63-94

14. Selladurai S, Muthukkumaran K, Kuppusami P, Divakar R, Mohandas E, Raghunathan VS (2007) Ionics 13:87-92

15. Muthukkumaran K, Kuppusami P, Mathews T, Mohandas E, Selladurai S Published online on Materials Science-Poland

16. McBride JR, Hass KC, Poindexter BD, Weber WH (1994) J Appl Phys 76(4):2435-2441

17. Goodenough JB (1995) Pure Appl Chem 67(6):931-938

18. Inaba H, Tagawa H (1996) Solid State Ion 83(1-2):1-16

19. Kosacki I, Suzuki T, Anderson HU, Colomban P (2002) Solid State Ion 149(1-2):99-105

20. Nomura K, Mizutani Y, Kawai M, Nakamura Y, Yamamoto O (2000) Solid State Ion 132(3-4):235-239

21. Zhang F, Chan S-W, Spanier JE, Apak E, Jin Q, Robinson RD, Herman IP (2002) Appl Phys Lett 80(1):127-129

22. Kosacki I, Suzuki T, Anderson HU, Colomban P (2002) Solid State Ion 149(1-2):99-105

23. Wang S, Wang W, Zuo J, Qian Y (2001) Mater Chem Phys 68 (1-3):246-248 